\title{
Passive impedance control of a multi-DOF VSA-CubeBot manipulator
}

\author{
Michele Mancini $^{\dagger}$, Giorgio Grioli ${ }^{\dagger}$, Manuel G. Catalano ${ }^{\dagger \ddagger}$, Manolo Garabini ${ }^{\dagger}$, Fabio Bonomo $^{\dagger}$ \\ and Antonio Bicchi ${ }^{\dagger \ddagger}$
}

\begin{abstract}
This work presents an example of the application of passive impedance control of a variable stiffness manipulator, which shows the actual benefits of variable stiffness in rejecting disturbances without resorting to the closure of a high level feedback loop. In the experiment a 4-DOF manipulator arm, built with the VSA-CubeBot platform, is controlled to hold a pen and draw a circle on an uneven surface. The control is designed calculating joint and stiffness trajectories with a Cartesian approach to the problem, thus designing the optimal workspace stiffness at first. Then, the joint stiffness yielding the closest workspace stiffness is searched for. Experimental results are reported, which agree with the theoretical outcomes, showing that the sub-optimal joints stiffness settings allow the arm to follow the circular trajectory on the uneven surface at best.
\end{abstract}

Index Terms-Physical Human-Robot Interaction, Performance, Variable Stiffness Mechanisms, Actuators, Robot, Multi DOF Robots, Modular Robots, Humanoid, Workspace Stiffness

\section{INTRODUCTION}

To control the interaction between a robotic manipulator and the environment is a crucial aspect for the successful execution of a wide number of tasks where the robot end-effector has to manipulate an object or perform some operations in contact with the environment, a problem generally referred to as constrained motion. In such cases, the use of a purely position-control oriented strategy for controlling interaction is candidate to fail. A solution to this was proposed, among others, in [1] and [2], which is called impedance control. The first implementation of this solution, active impedance control, usually requires force and/or torque measurements feedback and high speed sensors and controllers, to achieve sufficient bandwidth. One drawback of this strategy, shown in [3] is that, notwithstanding the low latencies achievable, the lag intrinsic in control can lead to behaviors which are unsafe in case of accidental impacts.

Passive impedance control has been proposed in [4] to overcome this kind of problems. The proposed solution is an actuator which allows for its mechanical impedance to be varied through an adequate transmission mechanism. A declination of this strategy is variable stiffness, where only the elastic part of the impedance can be varied. This topic has been widely treated and investigated in many works as illustrated in [5]. A large number of 1-DOF variable stiffness mechanisms has been studied and realized. Recently, few M-DOF systems have been built ([6], [7]). Work [7], in particular, presents a customizable platform for the realization and test of variable stiffness robotic structures

\footnotetext{
$\dagger$ Centro Int. di Ricerca "E. Piaggio", Univ. of Pisa, 56126 Pisa, Italy

$\ddagger$ Italian Inst. of Technologies, Adv. Robotics, 16163 Genova, Italy

\{michele.mancini, giorgio.grioli,

manolo.garabini, manuel.catalano

fabio.bonomo, bicchi\}ecentropiaggio.unipi.it
}

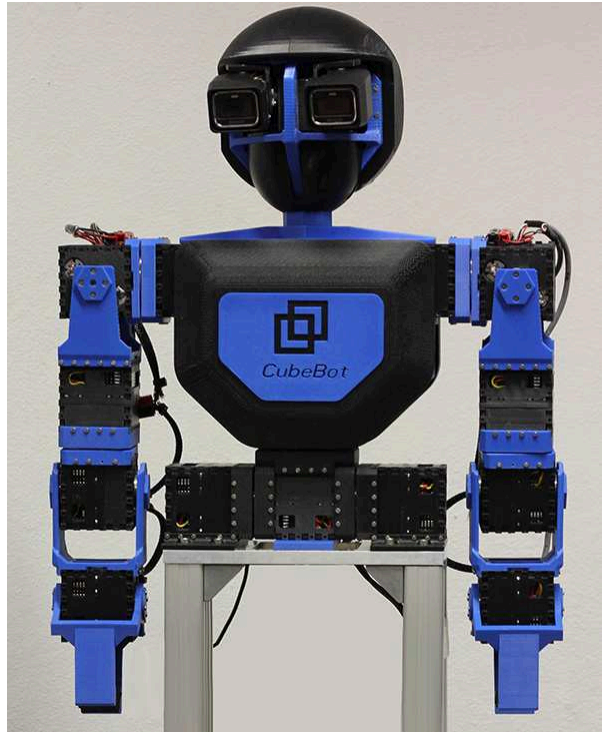

Fig. 1. Humanoid torso built using the VSA-CubeBot platform. Each arm is composed by four VSA-Cube modules plus one for the gripper. Two modules are used for the bending of the torso and another one is used for the rotation.

with many degrees of freedom (DOF), called VSA-CubeBot . A humanoid torso, built using this platform, is shown in Figure 1.

This article proposes an application of passive impedance control and presents an experiment to show the actual benefits of passive impedance control in the rejection of disturbances, as an alternative to the closure of an high level feedback loop. In particular, passive impedance control is used as a low complexity control system in contrast with active impedance control, suggesting for an alternative option with respect to what is considered in [8], where the tradeoff between the complexity of implementing a control law and the performance of the control system was already considered. Our approach is also an alternative to techniques of combined compliance control, as those proposed in [9] and [10], where the active part of the stiffness is purportedly neglected, to avoid any high-level feedback.

The control is designed defining the reference trajectory in the space of joints angles and stiffness, such to minimize a functional of the expected error along the trajectory. The control is, then, fed in open-loop to the actuator units, which, acting as servos, take care of implementing the trajectory and stiffness references. This approach is applied to the particular problem of drawing a circle on a wavy surface using the arm of the VSA-CubeBot robotic torso, holding a felt-tip pen. A rendering of the experimental setup is shown in Figure 2.

The problem of experiment design is stated in section II. 


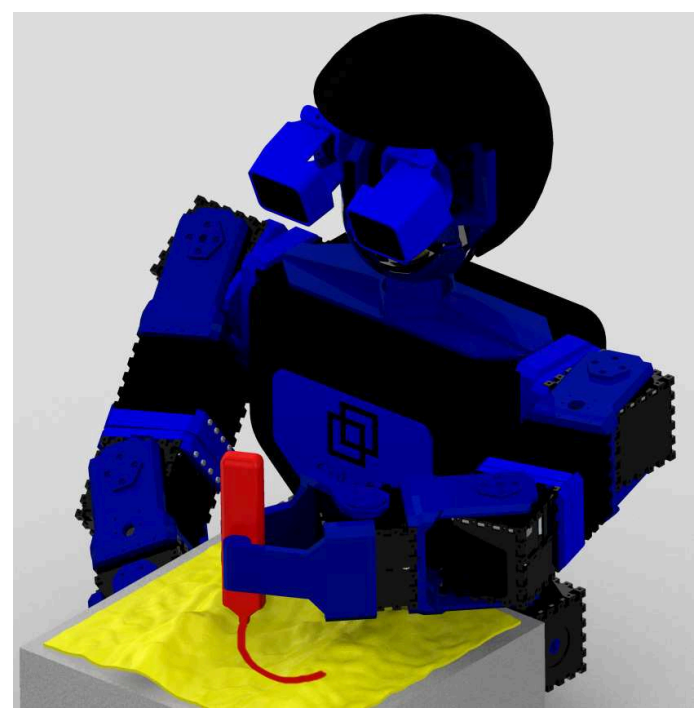

Fig. 2. CG render of the experimental setup.

It is divided in two parts, treated in sections III and IV. First the optimal workspace stiffness matrix is sought. Then an appropriate joints stiffness is searched for. In sections $\mathrm{V}$ and VI the setup and the results of the experiment are presented.

\section{PROBLEM STATEMENT}

Given a serial manipulator with variable stiffness joints, the problem of designing its control is not trivial, due to the high number of degrees of freedom on one side, which is the double of those of an equivalent rigid manipulator, and due to the increased complexity added by the presence of elasticity between the actuators and the robot links.

The second aspect of the problem can be faced drawing inspiration from solutions such as those of [11], which can be extended to variable stiffness. On the other hand, the possibility to manage the stiffness of the robot adds a totally different perspective to the design of the manipulator control. Approaches to this problem are present in literature, and range from the classical work [1] on Impedance Control, to more recent bio-inspired algorithms [12].

We propose a simple yet effective way to face this problem, which is based on the analysis of the task in the Cartesian space. The problem of the determination of a reference joint trajectory from a chosen task described in the Cartesian space is easily obtained inverting the kinematics of the arm with one of many traditional techniques (see for example [13] for a review).

In order to design the joints stiffness, an optimal problem is set-up in the Cartesian space at first. Considering the manipulator as a generalized translation spring that interacts with the environment via a single contact point, the relationship

$$
\mathbf{F}=\boldsymbol{\Sigma} \boldsymbol{\Delta}
$$

holds, where $\mathbf{F} \in \mathbb{R}^{3}$ is the vector of generalized force at the end-effector, $\Delta \in \mathbb{R}^{3}$ is the displacement of the end-effector from the reference position and $\boldsymbol{\Sigma} \in \mathbb{R}^{3 \times 3}$ is the stiffness matrix.

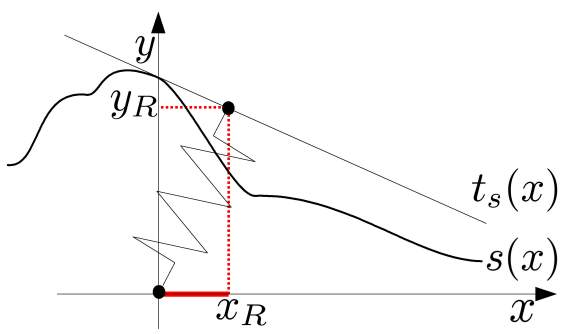

Fig. 3. Two-dimensional scheme of the workspace stiffness problem. The desired point is the origin of the Cartesian plane, $x_{R}$ and $y_{R}$ are the coordinates of the real end-effector's position, constrained on the tangent line $t_{s}$ that approximates the surface $s$. The $x$-axis is the in-plane direction and the $y$-axis is the normal direction. The target is to minimize the red solid line along $x$-axis, that is the projection, on the supporting plane, of the position error.

Defining a goal in the Cartesian space, in the form of a functional $J(x)$ (where $x$ is the end-effector position), an optimal stiffness matrix $\boldsymbol{\Sigma}_{d}$ can be found and then mapped into the joint stiffness through the congruence transformation (CT)

$$
\mathbf{K}=\mathbf{J}^{T} \boldsymbol{\Sigma} \mathbf{J},
$$

proposed in [14], which connects the joints stiffness and workspace stiffness using the Jacobian matrix $\mathbf{J}$ of the manipulator ${ }^{1}$.

The desired joint stiffness matrix $\mathbf{K}_{d}$ will then be approximated by a VSA robot using only its passive stiffness capabilities.

The most interesting aspect of a control designed in this way is the possibility to provide it completely in feedforward to the actuator units, yet obtaining satisfactory performance and intrinsic passivity (due to the lack of a feedback action).

The proposed technique will be applied now to the simple case of a robotic arm demanded to trace a circle on a surface with unknown irregularities minimizing the error between the desired trajectory and the projection of the executed trajectory on the supporting plane of the irregular surface. To this extent we will assume that the manipulator joints controls are the equilibrium position and the joint stiffness only.

\section{OPTIMAL WORKSPACE STIFFNESS}

In this section we design an optimal form of $\boldsymbol{\Sigma}$ allowing for the end effector to draw along a desired trajectory on an unknown wavy surface so that the projection of the trajectory on the supporting plane is not affected by the unevenness of the surface.

Let's look, for simplicity, to the problem reduced to a twodimensional case: referring to Figure 3 , the $x$-axis lies on the ideal (bump-less) drawing surface while the $y$-axis is on the normal direction. The unknown uneven surface is defined by the function $s(x)$. In a given instant of the trajectory, let the reference point be the origin of the reference frame $(0,0)$.

\footnotetext{
${ }^{1}$ Even if [15] proves that the CT is only valid at unloaded position, $\mathrm{CT}$ is used in place of the correct conservative congruence transformation (CCT) proposed in [16] for sake of simplicity (a study adopting CCT would need to acknowledge for the generally unknown load forces, and is demanded to future work).
} 
The equilibrium position $x^{*}$ of a point lying on the surface and attached to the origin by a general spring

$$
\boldsymbol{\Sigma}=\left[\begin{array}{ll}
\sigma_{x} & \sigma_{c} \\
\sigma_{c} & \sigma_{y}
\end{array}\right]
$$

can be found as:

$$
\min _{x} \mathcal{U}_{e l} \quad \text { subject to } \quad y=s(x)
$$

where $\mathcal{U}_{e l}$ is the elastic energy stored in the spatial spring. The cost function elastic energy $\mathcal{U}_{e l}$ is defined as:

$$
\mathcal{U}_{e l}=\frac{1}{2} \boldsymbol{\Delta}^{T} \boldsymbol{\Sigma} \boldsymbol{\Delta} \simeq \frac{1}{2}[x \beta+\gamma]^{T} \boldsymbol{\Sigma}[x \beta+\gamma]
$$

where

$$
\beta=\left(\begin{array}{c}
-1 \\
\alpha
\end{array}\right) \quad \gamma=\left(\begin{array}{c}
0 \\
s_{0}
\end{array}\right),
$$

and the last equality of 5 is obtained approximating the surface with its tangent line $t_{s}$, with slope $\alpha$ and intercept $s_{0}$. The equilibrium position is

$$
x^{*}=-\frac{\beta^{T} \boldsymbol{\Sigma} \gamma}{\beta^{T} \boldsymbol{\Sigma} \beta}=\frac{s_{0}\left(\sigma_{c}-\alpha\right)}{\sigma_{x}+\alpha^{2}-2 \alpha \sigma_{c}} .
$$

Scaling the matrix $\boldsymbol{\Sigma}$ by a constant factor, it can be noticed that the same result is achieved. In fact, defining

$$
\left\{\begin{array}{l}
\frac{\sigma_{x}}{\sigma_{y}}=\lambda_{x} \\
\frac{\sigma_{c}}{\sigma_{y}}=\lambda_{c}
\end{array} \Rightarrow \boldsymbol{\Sigma}=\sigma_{y}\left[\begin{array}{cc}
\lambda_{x} & \lambda_{c} \\
\lambda_{c} & 1
\end{array}\right],\right.
$$

the equilibrium point can be simplified as

$$
x^{*}=\frac{s_{0} \sigma_{y}\left(\frac{\sigma_{c}}{\sigma_{y}}-\alpha\right)}{\sigma_{y}\left(\frac{\sigma_{x}}{\sigma_{y}}+\alpha^{2}-2 \alpha \frac{\sigma_{c}}{\sigma_{y}}\right)}=\frac{s_{0}\left(\lambda_{c}-\alpha\right)}{\lambda_{x}+\alpha^{2}-2 \alpha \lambda_{c}} .
$$

Notice now that $x^{*}$, the position of the point on the surface $s$, can be used as a measure of the drawing error from the desired behavior, that is to draw an image that, viewed from above, is still a circle. In particular we want to minimize this error independently of the parameter $\alpha$ (the slope of the surface in a boundary of the desired point). Therefore we define the cost function $J\left(\lambda_{x}, \lambda_{c}\right)$ as the integral of the square error for every possible $\alpha$ :

$$
J\left(\lambda_{x}, \lambda_{c}\right)=\int_{-\infty}^{\infty} x^{* 2}(\alpha) d \alpha .
$$

This leads to the minimum problem:

$$
\min _{\lambda_{x}, \lambda_{c}} J\left(\lambda_{x}, \lambda_{c}\right) \text {. }
$$

Given the fact that $\lim _{\alpha \rightarrow \infty} x^{*}=0$, the integral 10 can be symbolically evaluated as

$$
J\left(\lambda_{x}, \lambda_{c}\right)=\frac{\pi}{2} \frac{s_{0}^{2}}{\sqrt{\lambda_{x}-\lambda_{c}^{2}}} .
$$

In Figure 4 the plot of the cost functional $J\left(\lambda_{x}, \lambda_{c}\right)$ is displayed. It is possible to see that the minimum is toward infinity for $\lambda_{x}$ and toward zero for $\lambda_{c}$. The analytical solution of this problem requires that all partial derivatives of $J$ with respect to $\lambda_{x}, \lambda_{c}$ must be zero at the same time.

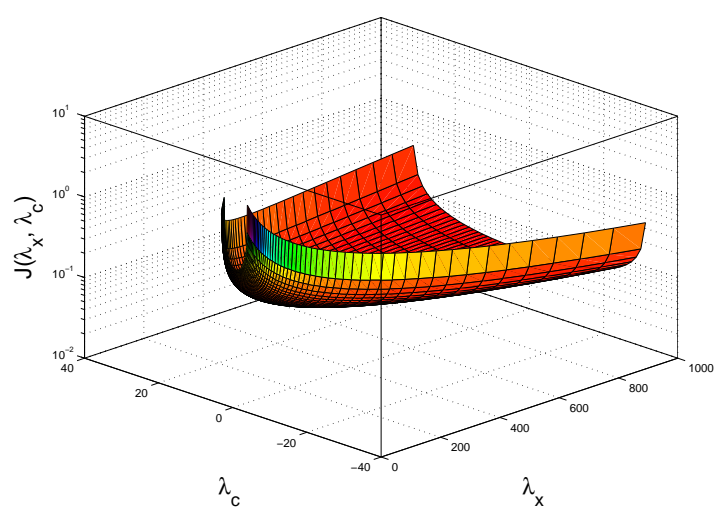

Fig. 4. Plot of the cost functional with respect to the elements of the stiffness matrix.

Those conditions are expressed by the following system of equations.

$$
\left\{\begin{array}{l}
\frac{\partial J}{\partial \lambda_{x}}=-\frac{\pi}{4} \frac{s_{0}^{2}}{\left(\lambda_{x}-\lambda_{c}^{2}\right)^{\frac{3}{2}}}=0 \\
\frac{\partial J}{\partial \lambda_{c}}=\frac{\pi}{2} \frac{s_{0}^{2} \lambda_{c}}{\left(\lambda_{x}-\lambda_{c}^{2}\right)^{\frac{3}{2}}}=0
\end{array}\right.
$$

Observing the system 13 can be asserted that both expressions tend to zero if $\lambda_{x} \rightarrow \infty$ and $\lambda_{c}$ has a finite value. While the second equation is true if $\lambda_{c}$ is zero.

If a set of constraints of the form

$$
\left(\begin{array}{c}
\sigma_{\min } \\
\frac{\sigma_{\min }}{\sigma_{\max }} \\
0
\end{array}\right) \leq\left(\begin{array}{c}
\sigma_{y} \\
\lambda_{x} \\
\lambda_{c}
\end{array}\right) \leq\left(\begin{array}{c}
\sigma_{\max } \\
\frac{\sigma_{\max }}{\sigma_{\min }} \\
\frac{\sigma_{\max }}{\sigma_{\min }}
\end{array}\right)
$$

is given, the optimal $\Sigma$ can be found in

$$
\boldsymbol{\Sigma}=\sigma_{\min }\left[\begin{array}{cc}
\frac{\sigma_{\max }}{\sigma_{\min }} & 0 \\
0 & 1
\end{array}\right]
$$

It is important to remark that a free parameter $\sigma_{\min }$ is there, meaning that the optimum can be reached for every matrix shaped as 15 .

This result can be extended to a trajectory in a $3 d$ workspace. The form of the obtained stiffness matrix follows the intuition: the end-effector should be (infinitely) stiff in the direction parallel to the plane and (infinitely) compliant in the normal direction, with no cross-interaction between the planar directions and the vertical one, as in

$$
\boldsymbol{\Sigma}_{\mathbf{d}}=\left[\begin{array}{cc|c}
\sigma_{\max } & * & 0 \\
* & \sigma_{\max } & 0 \\
\hline 0 & 0 & \sigma_{\min }
\end{array}\right]
$$

This formula can be rewritten to highlight the free parameter:

$$
\boldsymbol{\Sigma}_{\mathbf{d}}=\sigma_{\min }\left[\begin{array}{cc|c}
\frac{\sigma_{\max }}{\sigma_{\min }} & * & 0 \\
* & \frac{\sigma_{\max }}{\sigma_{\min }} & 0 \\
\hline 0 & 0 & 1
\end{array}\right] .
$$




\section{OPTIMAL JOINT STIFFNESS}

Given the manipulator used, hardware limits are such that the feasible joint stiffness is a diagonal matrix $\mathbf{K}(\mathbf{x}) \in$ $\mathbb{R}^{N \times N}$, where $N$ is the number of joints and $\mathbf{x} \in \mathbb{R}^{N}$ is the vector collecting the elements of the diagonal. The joint stiffness, producing a workspace stiffness of the manipulator as close as possible to the desired one, is found in this section.

This problem can be formalized as follows:

$$
\min _{\mathbf{x}} F(\mathbf{x}) \quad \text { subject to } \quad \mathbf{b}_{\mathbf{l}} \leq \mathbf{x} \leq \mathbf{b}_{\mathbf{u}} \quad
$$

where $F(\mathbf{x})$ is a cost functional that evaluates the distance between the actual joint stiffness and the desired joint stiffness $\mathbf{K}_{\mathbf{d}}$. The latter is calculated, using the CT (2), from the desired workspace stiffness $\boldsymbol{\Sigma}_{\mathbf{d}}$ found in section III. A generic configuration of the joints coordinates $\mathbf{q}$ and the knowledge of the position Jacobian $\mathbf{J}(\mathbf{q})$ are assumed. $\mathbf{K}_{\mathbf{d}}$ is a constant with the previous assumptions. The bounds $\mathbf{b}_{\mathbf{l}}$ and $\mathbf{b}_{\mathbf{u}}$ take into account the physical limits of the actuators.

To define a suitable cost functional $F(\mathbf{x})$, remember that, by virtue of 15 , the desired workspace stiffness is defined up to one degree of freedom. Because of the model CT, mapping $\boldsymbol{\Sigma}_{\mathbf{d}}$ in the $\mathbf{K}_{\mathbf{d}}$, is a linear operation, the set of admissible solutions in the space of the joint stiffness is defined by a line

$$
\tilde{\mathbf{K}}_{\mathbf{d}}=\mathbf{J}^{T} \sigma \boldsymbol{\Sigma}_{\mathbf{d}} \mathbf{J}=\sigma \mathbf{K}_{\mathbf{d}}
$$

which can be easily managed using the vectorized representation $\operatorname{vec}\left(\mathbf{K}_{\mathbf{d}}\right)$. To minimize the distance of $\operatorname{vec}(\mathbf{K}(\mathbf{x}))$ from the line of target solutions, we proceed to minimize the secant of the angle $\beta$ (as defined in Figure 5).

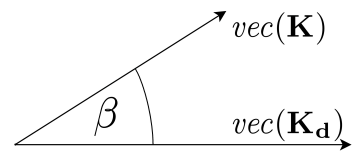

Fig. 5. Vectors representing the desired $K_{d}$ and the actual stiffness matrix of the joints $K$. The angle included between the vectors is minimum when they are parallel.

The secant of the angle $\beta$, comprised between the two vectorized stiffness, is minimum when they are aligned. Since $v_{1} \bullet v_{2}=\left\|v_{1}\right\|\left\|v_{2}\right\| \cos \widehat{v_{1} v_{2}} \quad \forall v_{i} \in V$ vector space,

$$
F(\mathbf{x})=\frac{\|\mathbf{x}\|}{\operatorname{vec}\left(\mathbf{K}_{\mathbf{d}}\right)^{T} \operatorname{vec}(\mathbf{K}(\mathbf{x}))}
$$

is proportional to $\sec \beta$, thus it expresses an angular distance.

This optimization problem can be solved numerically and would yield a trajectory in the space of joint stiffness which, in general, can be expressed as a function of the points of the trajectory.

\section{EXPERIMENTAL SETUP}

The experimental setup consists of the arm of a humanoid, realized by the VSA-CubeBot platform (Figure 1) and a wavy surface as shown in Figure 2. The surface, represented in Figure 6, is realized with ABS plastic and covered with strips of paper-tape.

\begin{tabular}{ccccc}
$\mathbf{n}$ & $\alpha$ & $\mathrm{a}$ & $\theta$ & $\mathrm{d}$ \\
\hline 1 & $-\pi / 2$ & 0 & $q_{1}$ & 0 \\
\hline 2 & $\pi / 2$ & 0 & $q_{2}$ & 0 \\
\hline 3 & $-\pi / 2$ & 0 & $q_{3}$ & $160 m m$ \\
\hline 4 & 0 & $170 m m$ & $q_{4}$ & 0 \\
\hline
\end{tabular}

TABLE I

MANIPULATOR's DENAVIT-HARTENBERg TABLE.

It is characterized by a maximum waviness height ${ }^{2}$ of $W_{h}=11.7 \mathrm{~mm}$ and a waviness spacing ${ }^{3}$ of $W_{s}=28.6 \mathrm{~mm}$.

The arm is made of 4 rotational joints with perpendicular rotation axes. The first 3 joints behave as a spherical wrist. The last joint determines the distance of the hand from the center of the shoulder. A $5^{t h}$ joint actuates a gripper. The Denavit-Hartenberg parametric description of the manipulator's kinematics is presented in Table I.

The arm is realized with VSA-Cube modules acting as servo-VSA: digital references of equilibrium positions and stiffness presets are commanded to each module and the low level logic inside implements a decentralized position and stiffness loop. No high level control feedback is used, the

\footnotetext{
${ }^{2}$ Waviness height is the height from the top of the peak to the bottom of the trough.

${ }^{3}$ Waviness spacing is the average spacing between waviness peaks.
}

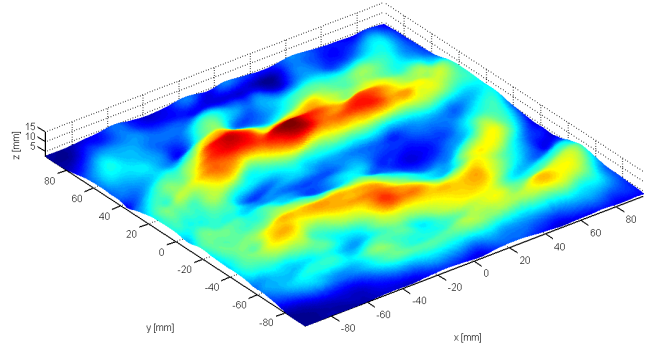

(a) 3D view

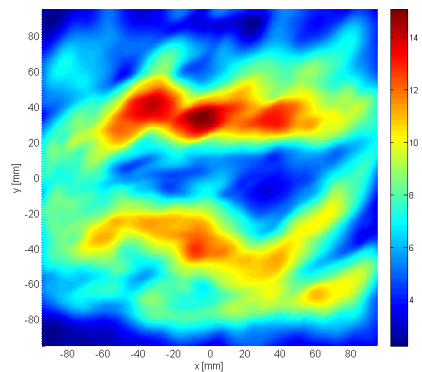

(b) xy view

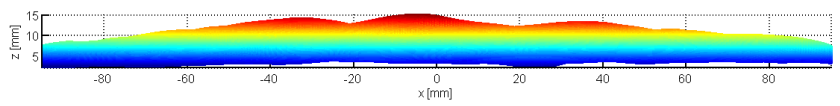

(c) $\mathrm{xz}$ view

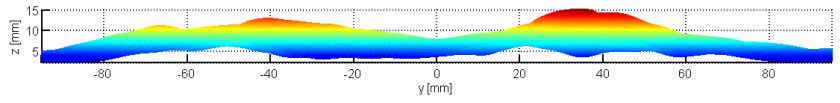

(d) yz view

Fig. 6. MATLAB plots of the irregular surface used for the experiment. 


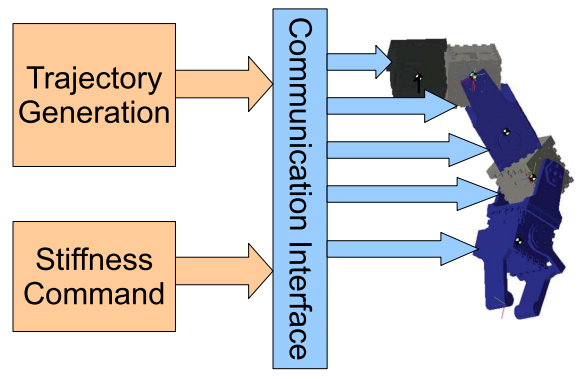

Fig. 7. Logic diagram of the command signals flow in the arm control system.

planned position and stiffness trajectories are fed directly to the actuators. The appropriate preset corresponding to a desired stiffness is calculated using the characterization data provided with the datasheets of the VSA-Cube modules. The command interface is realized in Matlab-Simulink, the signals flow is presented in Figure 7.

The circle has a radius of $45 \mathrm{~mm}$ on the plane $y_{0}=$ $0 \mathrm{~mm}$ (the horizontal plane containing the shoulder) and the coordinates of the circle center in the base frame are $p_{c}=\left[\begin{array}{lll}265 & 0 & 0\end{array}\right]^{T} \mathrm{~mm}$. A felt-tip pen is grasped with the gripper at the end of the arm and is kept perpendicular to the surface as if it (the surface) were flat. The felt-tip of the pen has a radius of $6 \mathrm{~mm}$.

The design problem was solved discretizing the calculated trajectory in a finite set of points and, then, solving the optimization problem with the Matlab function fmincon(). Since the values of the bounds $b_{l}$ and $b_{u}$ used are the minimum and maximum achievable stiffness of VSA-Cube modules: from $3,35[\mathrm{Nm} / \mathrm{rad}]$ to $11,59[\mathrm{Nm} / \mathrm{rad}]$, the result of the optimization indicates to set the $1^{\text {st }}$ and $3^{\text {rd }}$ joints to minimum stiffness, the $2^{\text {nd }}$ and the $4^{\text {th }}$ joint to maximum stiffness in every point of the circular trajectory.

Three test were performed with different values of joints stiffness. In trial I all the joints are set to high stiffness. This should replicate the behavior of a position controlled traditional robotic arm. In trial II the stiffness of all the joints is decreased to the minimum level imitating the behavior of a SEA-actuated robot ([17]). In trial III the second and fourth joints are set to the highest values, the first and third joints to the lowest values.

\section{EXPERIMENTAL RESULTS}

Figure 11 shows the projection of the end-effector trajectories on the support plane for the three experimental conditions, while Figure 12 reports mean square of the tracking error over time (corresponding to $J(\mathbf{x}) / t$ ). Figures 8,9 and 10, are pictures taken during the experiment.

Results of trial I indicate that, if the reference trajectory is high on the surface level, the surface is just skimmed, thus the circle is not completely drawn. If the reference trajectory is lowered the resistance of the arm on the bumps produces high reaction forces which causes the movement of the surface's support or the failure of the grip (Figure 8).

In trial II the arm leans on the surface and is not able to overcome the highest waves. As a consequence of this, the trajectory is severely deformed, as shown in Figure 9.

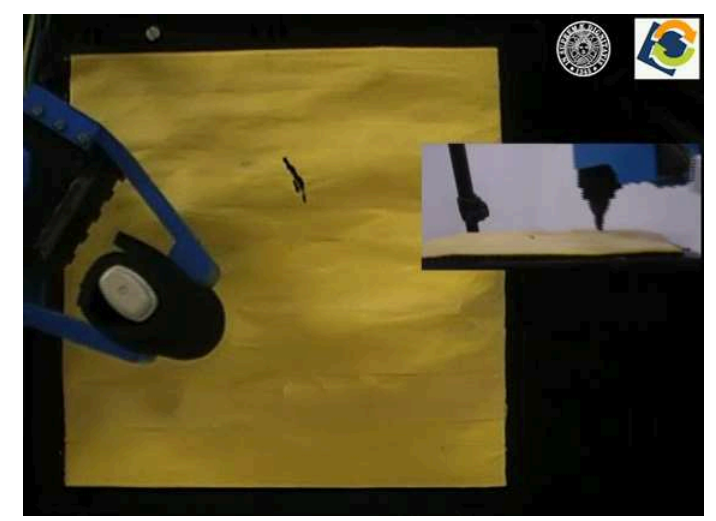

Fig. 8. Drawn trajectory on the wavy surface while all the joints are set to high stiffness. The surface is just skimmed, thus the trajectory is not traced completely and if the plane is raised the arm forces against the waves and moves the whole support or the grip is lost.

During trial III the in-plane forces are countered by the second and the fourth joints whereas the vertical forces are absorbed by the first and the third joints. The obtained drawing is much close to a perfect circle (Figure 10).

An interesting remark is that, within each trial the trajectory is repeated almost exactly at every cycle.

\section{CONCLUSIONS}

This work proposed a simple yet effective method for the design of the control of a Variable Stiffness robotic manipulator that enables the rejection of disturbances without resorting to closure of a high level control loop.

The end-effector stiffness is first designed looking at the problem in the Cartesian space and performing the optimization of a cost function. Then the desired Cartesian stiffness is mapped in the joint space, and approximated within the space of feasible joint stiffness matrices.

The method is validated applying it to the problem of drawing on a wavy surface with a 4-DOF VSA-CubeBot manipulator holding a felt-tip pen. A circular trajectory has been commanded to the robotic arm with three different settings for the joints stiffness: maximum rigidity on all joints, maximum compliance and finally the sub-optimal joint stiffness

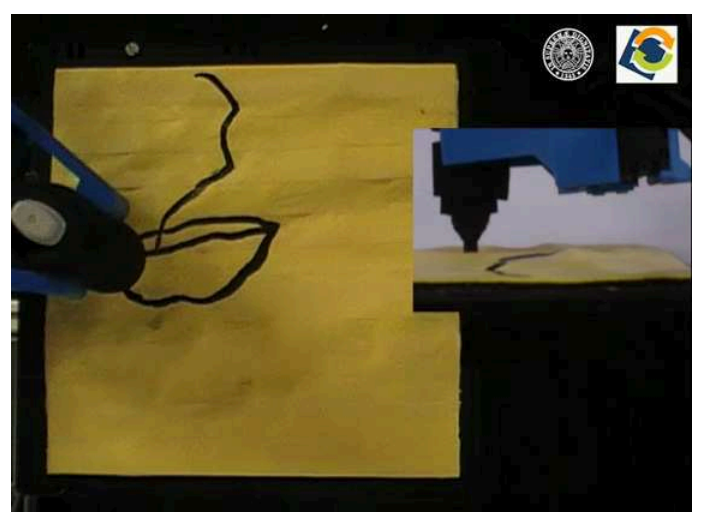

Fig. 9. Drawn trajectory on the wavy surface while all the joints are set to low stiffness. The arm leans on the surface and is not capable of going past the highest waves. The trajectory is severely deformed. 


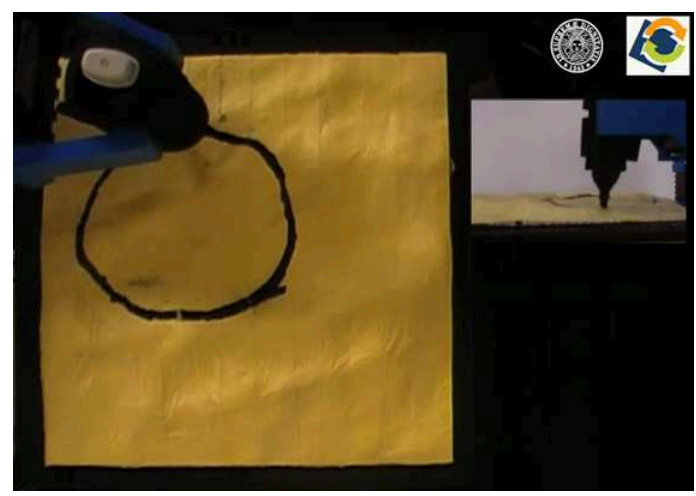

Fig. 10. Drawn trajectory on the wavy surface while the stiffness of each joint is set to the optimal value. The waves are absorbed by the more compliant joints and the trajectory is close to a circle.

found with our algorithm. The latter configuration yielded, as expected, the best drawing, while both the former two did not perform satisfactorily.

\section{ACKNOWLEDGMENTS}

Authors would like to gratefully acknowledge the useful work done by Fabrizio Vivaldi, Andrea di Basco, Niccoló Capecci, Enrico Gastasini and Riccardo Schiavi. A special thanks goes to Giovanni Tonietti. This work was partially supported by the VIACTORS, THE and ROBLOG Specific Targeted Research Projects, funded by the European Community under Contract ICT-231554-2009, ICT-248587-2010 and ICT-270350-2011, respectively.

\section{REFERENCES}

[1] N. Hogan, "Impedance control: An approach to manipulation," in American Control Conference, 1984, june 1984, pp. $304-313$.

[2] A. Albu-Schäffer, C. Ott, and G. Hirzinger, "A unified passivity-based control framework for position, torque and impedance control of flexible joint robots," Int. J. Rob. Res., vol. 26, pp. 23-39, January 2007. [Online]. Available: http://portal.acm.org/citation.cfm?id=1229555.1229558

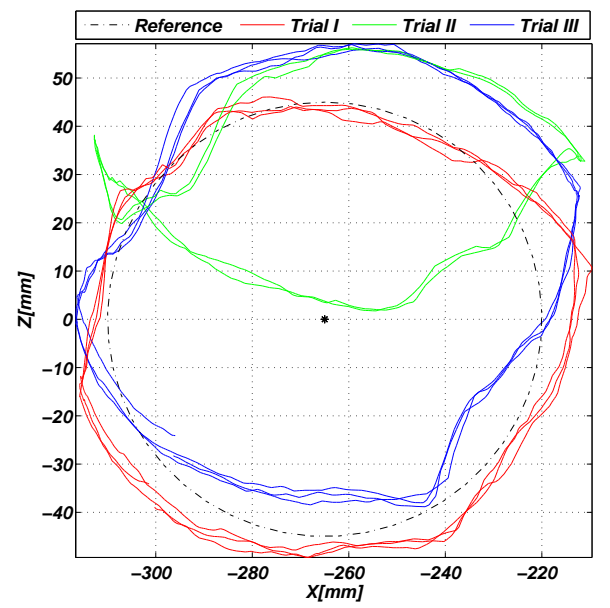

Fig. 11. Actual trajectory followed by the manipulator vs desired trajectory. Notice that the good tracking performances of trial I are just apparent, because the lack of compliance does not allow for continuous contact of the pen with the surface.

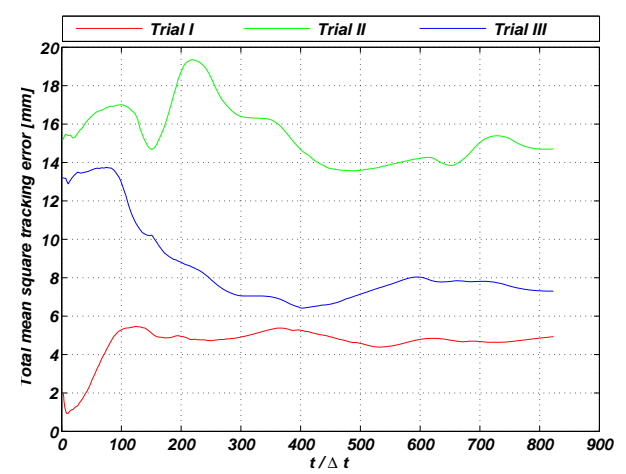

Fig. 12. Total mean square tracking error for the three experimental conditions. Notice that the good tracking performances of trial I are just apparent, because the lack of compliance does not allow for continuous contact of the pen with the surface.

[3] S. Haddadin, A. Albu-schffer, and G. Hirzinger, "Safety evaluation of physical human-robot interaction via crash-testing," in In Robotics: Science and Systems Conf. (RSS2007, 2007, pp. 217-224.

[4] A. Bicchi and G. Tonietti, "Fast and soft arm tactics: Dealing with the safety-performance trade-off in robot arms design and control," IEEE Robotics and Automation Magazine, vol. 11, no. 2, pp. 22-33, 2004.

[5] R. Ham, T. Sugar, B. Vanderborght, K. Hollander, and D. Lefeber, "Compliant actuator designs," Robotics Automation Magazine, IEEE, vol. 16 , no. 3, pp. $81-94$, september 2009.

[6] M. Grebenstein, A. Albu-Schaffer, T. Bahls, M. Chalon, O. Eiberger, W. Friedl, R. Gruber, S. Haddadin, U. Hagn, R. Haslinger, H. Hoppner, S. Jorg, M. Nickl, A. Nothhelfer, F. Petit, J. Reill, N. Seitz, T. Wimbock, S. Wolf, T. Wusthoff, and G. Hirzinger, "The dlr hand arm system," in Robotics and Automation (ICRA), 2011 IEEE International Conference on, may 2011, pp. $3175-3182$.

[7] M. G. Catalano, G. Grioli, M. Garabini, F. Bonomo, M. Mancini, N. Tsagarakis, and A. Bicchi, "Vsa - cubebot. a modular variable stiffness platform for multi degrees of freedom systems," in 2011 IEEE International Conference on Robotics and Automation, 2011.

[8] R. W. Brockett, "Minimum attention control," in Proc. 36th IEEE Conf. on Dec. and Control, San Diego, CA, 1997, pp. 2628-2632.

[9] K. Yokoi, H. Maekawa, and K. Tanie, "A method of compliance control for a redundant manipulator," in Intelligent Robots and Systems, 1992., Proceedings of the 1992 lEEE/RSJ International Conference on, vol. 3. IEEE, 1992, pp. 1927-1934.

[10] G. Palli and C. Melchiorri, "Interaction force control of robots with variable stiffness actuation."

[11] M. Spong, "On the force control problem for flexible joint manipulators," Automatic Control, IEEE Transactions on, vol. 34, no. 1, pp. $107-111,1989$

[12] K. Tee, D. Franklin, M. Kawato, T. Milner, and E. Burdet, "Concurrent adaptation of force and impedance in the redundant muscle system," Biological cybernetics, vol. 102, no. 1, pp. 31-44, 2010.

[13] B. Siciliano and O. Khatib, Springer handbook of robotics. SpringerVerlag New York Inc, 2008.

[14] J. K. Salisbury, "Active stiffness control of a manipulator in cartesian coordinates," in Decision and Control including the Symposium on Adaptive Processes, 1980 19th IEEE Conference on, vol. 19, 1980, pp. $95-100$.

[15] S.-F. Chen and I. Kao, "Simulation of conservative congruence transformation. conservative properties in the joint and cartesian spaces," in Robotics and Automation, 2000. Proceedings. ICRA 'OO. IEEE International Conference on, vol. 2, 2000, pp. 1283 -1288 vol.2.

[16] S.-F. Chen, Y. Li, and I. Kao, "A new theory in stiffness control for dextrous manipulation," in Robotics and Automation, 2001. Proceedings 2001 ICRA. IEEE International Conference on, vol. 3, 2001, pp. $3047-3054$ vol.3.

[17] G. A. Pratt and M. Williamson, "Series elastics actuators," in IROS, 1995, pp. 399-406. 